\title{
LIVER SINUSOIDAL ENDOTHELIAL CELL (LSEC) ISOLATION FOLLOWING A LIVER PERFUSION TECHNIQUE
}

Shahida Saharudin ${ }^{1}$, Norlelawati A. Talib ${ }^{1}$, Nor Zamzila Abdullah¹, Jamalludin Ab. Rahman $^{1}$ and Zunariah Buyong ${ }^{1}$

${ }^{1}$ Kulliyyah of Medicine, International Islamic University Malaysia, Kuantan, Pahang, Malaysia.

Presenter: Shahida Saharudin, shida_shs@iium.edu.my

Introduction: Liver perfusion has been the standard method to digest and isolate liver cells including liver sinusoidal endothelial cells (LSEC). Poor cannulating skills through portal vein results in a waste of animal resource. Familiarization of both liver perfusion technique and adhering strictly to aseptic technique during cell handling ensure high cell yield, minimum morphology disruption and cell contamination. We aimed to present a method of liver perfusion procedure followed by the isolation of LSEC.

Materials and method: The study was conducted with the approval of IACUC committee. Seven Sprague Dawley rats underwent these procedures under anaesthesia. Liver perfusion was done as previously described. Briefly, LSEC were isolated by liberase enzyme perfusion of the liver, isopycnic sedimentation in a two- step Percoll gradient and selective adherence. The purification and cultivation of LSEC was evaluated by light and electron microscopy.

Results: Purity and viability of LSEC after selective adherence was $80.5 \pm 3.5 \%$ and $\geq 95$ $\%$, respectively. The average concentration of the cells ranged from $32-75 \times 10^{6}$ per 400 gm rat. After 8 hours of culture, LSEC monolayers were contaminated with less than $5 \%$ of other cells.

Conclusion: This method is reliable and reproducible for the isolation of LSEC to enable the study of structure and function of these cells in vitro. However, improvement on the perfusion skills and isolation technique are vital to ensure better cell purity. 